CIENCIA Y SOCIEDAD

Volumen XXXII, Número 3

Julio-Septiembre 2007

\title{
COMPARACIÓN DE RASGOS DE PERSONALIDAD \\ ENTRE ESTUDIANTES DOMINICANOS DE 4TO. DE BACHILLERATO BILINGÜES Y MONOLINGÜES
}

\section{Abraham Barouch Gilbert* María Soledad Vargas* León Valeirón*}

\begin{abstract}
RESUMEN
Este fue un estudio comparativo con dos grupos: el Grupo A (40 sujetos) de personas bilingüies (Colegios bilingües) y el Grupo B( 40 sujetos ) de personas monolingües (Colegios monolingües). Los participantes de ambos grupos estaban equiparados en otras variables como: edad, sexo, nivel socio-económico y educativo.

Para fines de esta investigación la diferencia relevante, la variable independiente, fue el bilingüismo y la variable dependiente la personalidad. Se presume que el mismo influye en los rasgos de personalidad.

Para estimar las posibles diferencias entre los rasgos de personalidad de estos grupos se usó la prueba T de student.
\end{abstract}

PALABRAS CLAVE: Bilingüismo, rasgos de personalidad, actitudes adaptadas a la cultura, cambios culturales.

\section{ABSTRACT}

This is a comparative study with two groups of students: Group A (40 subjects) of bilingual people (bilingual Schools) and the Group B ( 40 subjects) of monolingual people (monolingual Schools). The participants of both groups were compared in other variables like: age, sex, socioeconomic and educative level.

For the objective of this investigation the important difference, that is, the independent variable, was the bilingualism and the dependent variable the personality. It is presumed that the first one influences in the personality characteristics. In order to consider the possible differences between the characteristics of personality of these groups we used the T test of Student.

KEY WORDS: Bilingualism, Characteristics of Personality, Attitudes Adapted to the culture, Cultural Changes.

* Psicología, Ciencias Sociales, Instituto Tecnológico de Santo Domingo, INTEC

E-mail: sociales@intec.edu.do 
En esta investigación fue de interés ver los rasgos de personalidad y su relación con el bilingüismo. Chipongian ( 2000 ) hace referencias a los primeros estudios acerca del bilingüismo y los mitos o los supuestos efectos negativos del bilingüismo, entre ellos señala los que siguen.

De acuerdo con Colin Baker, autor de "Foundations of Bilingual Education and Bilingualism", éste relaciona el bilingüismo con confusión cerebral, personalidad partida, hasta privación espiritual.

En el caso del bilingüe muchos de los estudios hablan de dos personalidades y esto se debe a los señalamientos que hace Ramírez Nairan et.al (2004) de (Grosjean 1982), "en este grupo se nota, incluso por lo mismos bilingües, que los bilingües expresan diferentes personalidades cuando hablan en diferentes idiomas".

Aquí se refiere a un individuo bicultural, y estos a su vez son aquellos que tienen internalizadas dos culturas y pueden guiar sus sentimientos, pensamientos y acciones. (Ramírez Nairan et.al 2004).

En ese estudio hecho por Ramírez Nairan et.al (2004) se vio claramente que los individuos bilingües demostraban diferentes tipos de actitudes y valores cuando respondían los mismos cuestionarios en diferentes idiomas.

También hay más estudios que si señalan que hay diferencias en la personalidad de los sujetos bilingües y explican que esto es un resultado de cambios culturales (cultural shifts). (Ramírez Nairan et.al 2004)

Estos estudios señalan diferencias entre los grupos, por ejemplo, los bilingües mostraban más eficiencia intelectual cuando respondían en ingles en vez de español.

Aquí se hace reseña a las actitudes que se adoptan de parte de las culturas que con el lenguaje se adquiere. En otras palabras, actitudes que en una cultura se adoptan y en otra no. (Ramírez Nairan et.al 2004).

En algunos casos se da el "code switching" este se entiende como la alternación de lenguajes dentro de una conversación. Estas alternaciones entre lenguajes pueden cambiar contexto o posición (Aver 1988) .(Palgrave).

Por ejemplo Sebba y Woottons (1998) reportan un estudio hecho de "code switching", el cual demostró como los bilingües usaban este cambio para construir identidades, un proceso negociado a través de recursos dentro y fuera de interacciones por sí mismos.(Palgrave). 
Finalmente un estudio de Ramírez Nairan et.al ( 2004 ) reporto puntuaciones de extroversión(socialmente desenvuelta, no inhibida, etc) consciente(responsable, perseverante, organizada, etc), afectotimia(afable, reposada, emocionalmente expresiva, bondadosa, adaptable, etc) más altas en los bilingües y los monolingüies puntuaron más alto en neurotiscismo (ansiedades, temores injustificados, etc).

Edwards (2004) señala que hay evidencia de que la preferencia por un lenguaje puede que implique diferentes aspectos de la personalidad. Sujetos bilingües que respondían a entrevistas y cuestionarios daban de manera muy ligera fotos diferentes de sí mismos dependiendo del lenguaje usado.

Los sujetos bilingües también pueden tener un sentido más fuerte de identidad en un idioma que en otro pero esto no implica personalidades separadas.

Hakuta (1991) señala la siguiente aportación de Peal (1962) "las experiencias de los bilingües son más amplias por las dos culturas y esto le da una ventaja que el monolingüe no tiene".

Benet (2005) resalta que los sujetos biculturales se mueven en sus dos orientaciones culturales mediante un cambio en la óptica basado en la cultura que responde a señales culturales.

En este mismo artículo se resalta un estudio hecho en University of Texas en Austin, en la cual se les preguntaba a bilingües (mexicano-americano) unas series de preguntas hechas para determinar personalidad. En las respuestas de estos individuos se vio claramente una variación y esto ocurría cuando se les cambiaban los idiomas.

Cuando estos respondían en inglés su respuestas enfatizaban más la asertividad y logros.

Estos rasgos encajaban con los ideales individualistas de los estadounidenses, opuesto a la cultura orientada en grupo de México. Esto lo explica la investigadora principal Nairan Ramírez -Esparza. (Aaronson 2006).

También hay cosas especificas universales y de la cultura que corresponden a esta variación en la personalidad. Algunos aspectos específicos responden a síndromes culturales, así como complejidades, individualismo y colectivismo. (Triandis 2002).

Cattell plantea en su libro "El Análisis Científico De La Personalidad" (1972) su punto de vista sobre el desarrollo de los rasgos por medio del aprendizaje. En uno de esos puntos señala el condiciona- 
miento clásico y el otro el condicionamiento operante $\mathrm{Y}$ agrega que mediante estos se pueden producir modificaciones en la conducta.

Se puede apreciar de manera muy clara que las lenguas van de la mano con las culturas y éstas a su vez están llenas de códigos conductuales los cuales se refuerzan constantemente y de ahí viene una especie de condicionamiento con el individuo y éste emite una conducta diferente dependiendo del lenguaje en uso.

Cattell (1972) también agrega que mediante las interminables aplicaciones de recompensas y castigos (privaciones)en la familia, colegio, y grupo de amigos se van formando gradualmente ciertos modelos de respuesta de la personalidad -rasgos-ADAPTADOS A LA CULTURA SOCIAL.

Por lo tanto cada lengua tiene sus modelos de respuesta de la personalidad y partiendo de allí se sobreentiende que el sujeto bilingüe sí recibe o hay algún tipo de incidencia por su segunda lengua que como resultado trae una modificación en la personalidad o una reestructuración de la misma.

Por ejemplo, en un artículo hecho por Aaronson (2006), ésta señala que el lenguaje es la madre del pensamiento y también es una fuente de personalidad.

También aporta que las personas difieren en rasgos de personalidad dependiendo en qué lenguaje hablen.

Una explicación potencial para los cambios dependientes del lenguaje observados en la personalidad de los bilingües se ve cuando estos sujetos cambian de un idioma a otro y cuando esto ocurre se da un cambio del marco cultural en el individuo. Por esta razón muchas veces se dice que los sujetos bilingües son s ujetos biculturales. (Ramirez Nairan et.al.2004).

Dentro de este planteamiento vale señalar como explicación de este fenómeno el concepto de acomodación cultural. Este se parece mucho al cambio de marco cultural, pero éste se ve claramente cuando un bilinguie responde a una situación dada de manera que acomoda o favorece la cultura asociada con el idioma en uso. El lenguaje se asocia con valores culturales, actitudes y memoria y cuando estos se activan influyen en la conducta. .(Ramirez Nairan et.al.2004). 
Llegando a la parte final se señala que el objetivo principal de esta investigación fue comparar los Rasgos de Personalidad entre estudiantes dominicanos de 4to de bachillerato de colegios bilingües y monolingües. Vale resaltar que solo dos factores son significativos (Factor M, IP) según el manual del test 16FP (1995) los demás puntuaron dentro de lo normal.

Viendo los resultados en promedio de los rasgos de la personalidad se puede inferir lo siguiente de cada grupo.

(Se hace la salvedad que la interpretación de los factores es tomada del manual del 16 FP, 1995 , Epsi)

\section{Sujetos Bilingües}

F- Los sujetos bilingües tienden a ser mas descuidados (confiados a la buena ventura, este tipo de persona tiende a ser jovial, activa, franca, expresiva. Frecuentemente se le escoge como líder electo.).

H- Son mas emprendedores, socialmente atrevidos, espontáneos, no inhibidos (Estos tienden a ser sociables, atrevidos, dispuestos a intentar nuevas cosas, espontáneos, de numerosas respuestas emociónales indiferencia le permite soportar sin fatiga el "toma y daca" del trato con la gente y las situaciones emocionales abrumadoras. Sin embargo puede despreocuparse por los detalles, e invertir mucho tiempo charlando.

I- Son más dependientes, impresionables. (Estos sujetos dejan afectarse por sus sentimientos, idealistas, soñadores, artistas. A veces solicitan para sí la atención y ayuda de otros, son impacientes, dependientes y poco prácticos.

L- Los sujetos bilingües son mas suspicaces, difíciles de engañar (Estos sujetos son desconfiados y ambiguos. A menudo se encuentran complicadas en su propio yo, les gusta opinar sobre sí mismos y están interesados en la vida mental íntima. Suelen actuar con premeditación. 
Q1- Son más analíticos, liberales, experimentales, de ideas libres, pensamientos abiertos (Estas personas suelen interesarse por cuestiones intelectuales y dudar de los principios fundamentales. Suele estar bien informada, poco inclinados a moralizar y más a preguntar por la vida en general y ser más tolerantes con las molestias y el cambio.

Q3- También son más controlados, socialmente adaptados, mucho control de su auto imagen. Estos sujetos suelen tener mucho control de sus emociones y conducta en general y tienden a ser cuidadosos y abiertos a lo social con "respeto hacia si mismos".

Q4- Son más tensos, frustrados, sobreexcitados (Su frustración representa un exceso del impulso de estimulación no descargado).

QI- Los sujetos bilingües en promedio según esta muestra son más Extrovertidos (son socialmente desenvueltos, no inhibidos, con buena capacidad para lograr y mantener contactos personales).

QII- Son mas ansiosos (en su sentido corriente) pero no son necesariamente neuróticos pues la ansiedad puede ser ocasional, pero puede presentar algún desajuste, como estar insatisfechos con su posibilidad de responder a las urgencias de la vida o con sus éxitos en lo que deseen.

QVII- Poco moralistas (son permisivos y poco propensos a juzgar a los demás por sus acciones).

IL- Los sujetos bilingües puntuaron mas alto en alto liderazgo para dirigir grupos, coordinar las funciones de los integrantes y prestar atención a los detalles y preocupaciones del grupo.

\section{Sujetos Monolingües}

A- Los sujetos monolingües son más abiertos, afectuosos, participativos (tienden a ser afables, dispuestos a cooperar, les gustan las ocupaciones que exijan contacto con la gente). 
Abraham Barouch Gilbert, María Soledad Vargas, Leo Valeirón: Comparación de rasgos...

C- Estos sujetos son emocionalmente mas estables, tranquilos, son realistas acerca de la vida, con buen firmeza interior y capacidad para mantener una sólida moral del grupo.

E- Estos sujetos tienden a ser más dominantes, independientes, agresivos, dogmáticos, seguros de si mismos, de mentalidad independiente, también autoritarios con el manejo de los demás).

M- Los sujetos monolingües son más imaginativos, centrados en sus necesidades intimas, abstraídos, despreocupados de los asuntos prácticos.

N- Estos son más astutos, calculadores. Su enfoque es intelectual y poco sentimental, aproximándose a las situaciones de una manera casi cínica.

Q2- También son más autosuficientes, prefieren sus propias decisiones. (Temperamentalmente independientes, acostumbran a seguir sus propios caminos).

Q3- Estos sujetos son más emprendedores, decididos y animados, a veces irreflexivos e ignoran las sutilezas de las relaciones.

Q4- Estos son independientes, prudentes y cuidadosos. También son más subjetivos, algo egocéntricos y emotivos.

IR- Los monolingües son más hábiles para desarrollarse apropiadamente en las situaciones de estudio o de trabajo intelectual, también son más creativos, artísticos e imaginativos.

\section{Puntuaciones iguales}

Los sujetos bilingües y monolingües puntuaron en promedio iguales cantidades en los siguientes factores:

IN: Emocionalmente estable, sin signos de desajuste neurótico. Parece poco propensa a reaccionar a los estímulos emocionales y no se preocupa excesivamente de los acontecimientos. 
IP: Coherente, realista con un espíritu practico y escasa tendencia a meditaciones o reflexiones de tipo espiritual. Parece ser dogmático, con intereses centrados en campos prácticos y realisticos.

A pesar de que las puntuaciones en promedio de los factores de ambos grupos según el manual del 16FP estaban dentro de lo normal se pudieron observar diferencias en casi todos los factores menos en los dos mencionados anteriormente. (Factor M, IP).

Se puede inferir que hay una ligera variación en los rasgos de personalidad en ambos grupos. Por ejemplo se vio que el grupo de los bilingües puntuó más alto en extroversión la cual es señalada en investigaciones hechas anteriormente por Ramírez Nairan et.al (2004) la cual reporto puntuaciones de extroversión (socialmente desenvuelta, no inhibida, etc.) consciente (responsable, perseverante, organizada, etc.), afectotimia (afable, reposada, emocionalmente expresiva, bondadosa, adaptable, etc.) más altas en los bilingües. Los monolingües puntuaron más alto en neurotiscismo (ansiedades, temores injustificados, etc.). Pero en nuestra población se vio que ambos grupos estaban dentro de lo normal en el factor IN (neurosis) y además de esto sus puntuaciones son iguales.

Por otro lado no se pueden tomar los resultados obtenidos en esta investigación como puntuaciones definitivas ya que hay otras maneras más claras en las cuales se pueden ver los diferentes rasgos o las asociaciones culturales con el lenguaje a evaluar.

Se retoma como ejemplo mencionado anteriormente el estudio hecho en University of Texas en Austin, en el cual se les preguntaba a bilingües (mexicano-americano) unas series de preguntas hechas para determinar personalidad. En las respuestas de estos individuos se vio claramente una variación y esto ocurría cuando se les cambiaban los idiomas.

Cuando estos respondían en ingles su respuestas enfatizaban más la asertividad y logros.

Estos rasgos encajaban con los ideales individualistas de los estadounidenses, opuestos a la cultura orientada en grupo de México. Esto lo explica la investigadora principal Nairan Ramírez -Esparza. (Aaronson 2006). 
Abraham Barouch Gilbert, María Soledad Vargas, Leo Valeirón: Comparación de rasgos...

Por lo tanto se señala que estos estudios sobre el lenguaje y la personalidad deben ser más complejos y profundos para así poder entender realmente con más claridad los procesos involucrados. 


\section{Referencias Bibliográficas}

1- APA press releases(2004). BEING BILINGUAL PROTECTS AGAINST SOME AGE-RELATED COGNITIVE CHANGES, SAYS NEW RESEARCH. American Psychological Association.

Disponible: http://www.apa.org/releases/bilingual_aging.html

2- Aaronson Lauren. Language my Spanish side. Psychologytoday.Enero 2006.

Disponible:http://www.psychologytoday.com/articles/pto20050617-000011.html

3- Benet Verónica .Bicultural Identity Intergration .Components abd Psychosocial Antecedents.Journal of Personality .73:4. Agust 2005.Blackwell Publishing.University of California at RiverSide.

4- Brain Connection. 15 de Febrero 2005

Disponible:http://www.brainconnection.com/nav/bcfnf.php3?Ur $\mathrm{i}=\% 2$ Ftopics $\% 2 \mathrm{~F} \% 3 \mathrm{Fmain} \% 3 \mathrm{Dfa} \% 2 \mathrm{Fsecond}$-languge $3 \& \mathrm{ref}=$.

5- Chipongian Lisa (2000) Brain Connection.

Disponible :http://www.brainconnection.com/topics/printindex. php3?main=fa/cognitive-bilingualism

6- Barnouw Victor. Culture and Personality.1963.The Dorsey Press. Homewood Illinois.

7- Birner Betty. Bilingualism . Linguistic Society of America.

Disponible : http://www.lsadc.org/info/ling-faqs-biling.cfm

8- C.A.B.E 2003 ) RedNova News : Children Raised Bilingually May Be Smarter . California Association for Bilingual Education, ( 2003).

Disponible:http://www.bilingualeducation.org/bilnews_n.html

9- Casado Manuel. Lenguaje y Cultura.(1988). Editorial Síntesis. Madrid.

10- Cattell Raymond El Análisis Científico de la Personalidad(1972).Editorial Fontanella. Barcelona. 
Abraham Barouch Gilbert, María Soledad Vargas, Leo Valeirón: Comparación de rasgos...

11- Chipongian Lisa. (2000) . The Cognitive Advantages of Balanced Bilingualism .

Disponible: http://www.medicalnewstoday.com/medicalnews. php?newsid $=9468$

12- Gumperz Jhon. Lenguaje y Cultura.( 1980).Editorial Anagrama.Barcelona.

13- Edwards John. Foundations of Bilingualism.2004.Bilingualism is belonging. Blackwell Publishing.

Disponible: www.catesol.org/Chung_Zhang.pdf

13- Georgetown University, Department of Psychology, T TEST, Research Methods and statitstics Resources.

Disponible:http://www.georgetown.edu/departments/psychology/researchmethods/statistics/inferential/ttest.htm

14- Fantini E. Alvino. La adquisición del lenguaje en un niño bilingüe.1982.editorial Herder. Barcelona.

15- Feldman S.Robert . Psicología con aplicaciones en países de habla hispana. 2001. Editora Ultra . Distrito Federal. 301.

16- Hakuta Kenji. ( 1991 ) Bilingualism as a Gift . Working Paper Series, No 33.

Disponible: http:// ccsre.stanford.edu/pdfs/wps33.pdf

17- Hakuta Kenji (1990) Bilingualism and bilingual education : a research perspective. NCBE FOCUS: Occasional Papers in Bilingual Education, Number 1.

Diponible : http://www.ncela.gwu.edu/pubs/focus/focus1.htm

18- Hakuta Kenji , Suben Judith ( 1985 ) Annual Reviews of Applied Linguistics.

Volume 6 Cambridge University Press . Disponible :

http://faculty.ucmerced.edu/khakuta/research/publications/(1985)\%20 \%20BILINGUALISM\%20AND\%20COGNITIVE \%20DEVELOPMENT.pdf 
19- Ho Yu Chong 2000. Disponible: http://www.creative-wisdom.com/pub/aera2000.html

8 de Marzo 2005.

20- Kim Karl H. S., Relkin Norman R, Lee Kyoung-Min \& Hirsch Joy, et .al. Distinct cortical áreas associated with native and second languages. Nature . 388 . 1997. 171-174

21- Lefrancois R Guy. El ciclo de la vida .2001. International Thomson Editores . Distrito Federal. 150-205.

22- Maceri Domenico, 2006 . Does Bilingualism Keep You Young?. The Seoul Times. Disponible: http://theseoultimes.com/ ST/?url=/ST/db/read.php?idx $=901$.

8de marzo 2005.

23- Mechelli Andrea, Crinion Jenny T., Noppeney Uta, et. al; Neurolinguistics: Structural plasticity in the bilingual brain. Nature. 431. 2004. 757.

15 de Febrero 2005.

24- Morris Charles G. UNDERSTANDING PSYCHOLOGY.1993.Prentice Hall. ENGLEWOOD CLIFFS, New Jersey.

25- Neve Alarcón Josefina Luisa. EL FENÓMENO DEL BILINGÜISMO Y SUS IMPLICACIONES EN EL DESARROLLO COGNITIVO DEL INDIVIDUO. (1998).

Disponible:http://www.uv.mx/iie/Coleccion/N_29/el_ fen\%C3\%B3meno_del_biling\%C3\%BCismo.htm

26- Papalia E . Diane, 2001. Psicología del Desarrollo. McGrawHill. Bogota

27- Pelgrave. Researching Multilingualism and Multilingual Identities. Disponible: www.palgrave.com/pdfs/1403939659.pdf

28- Prensa Grafica. Disponibl: Http://www.iin.oea.org/Noticias \%20pasadas\%202002-2003/Noticias\%20desplegadas\%20julio\%20 2003/noticia32b.htm. 15 de Febrero 2005. 
Abraham Barouch Gilbert, María Soledad Vargas, Leo Valeirón: Comparación de rasgos...

29- Queensland Health . 2 December 2002.

Disponible : http://www.health.qld.gov.au/abios/tbi/bparieta.asp

30- Raven J.C. J . Raven . J.H. Court. Test de Matrices Progresivas Manual. 2004. Editorial Paidos .Buenos Aires .

31- Ramirez Nairan. et al. Do bilinguals have two personalities? A special case of cultural frame switching. Journal of Research in Personality (2004). The University of Texas.

32- Rodríguez-fornells Antoni, Rotte Michael, Heinze Hansjochen, et. sl. Brain potential and functional MRI evidence for how to handle two languages with one brain. Nature . 415 .2002 . 10261029. 17 de Febrero 2005.

33- Salkind ,Neil J. Metodos de Investigación.1999.Prentice Hall .México.

34- Spreen Otfried, Ester Strauss . A compendium of Neuropsychological Tests. 1998. Oxford University Press, Inc. New York . 83-89. 11 de Febrero

35- Swan Michael, Walther Catherine .Test yourself.1997. Oxford University Press. Oxford New york.(ENGLISH TEST).

36- Thymos . Disponible : Http://www.thymos.com/science/language.html . 15 de Febrero 2005.

37- Triandis Harry. CULTURAL INFLUENCES ON PERSONALITY. 1Department of Psychology, University of Illinois, Champaign-Urbana, Champaign, Illinois. Vol. 53: 133-160 (Volume publication date February 2002).

Dsiponible:http://arjournals.annualreviews.org/doi/abs/10.1146/ annurev.psych.53.100901.135200; jsessionid=iuyx_NRQ7Zd9x6H $\mathrm{mSM}$ ?cookieSet $=1 \&$ journalCode $=$ psych

38- Universidad Pedagógica Nacional. Febrero 14,2005

Disponible:Http://interbilingue.ajusco.upn.mx/modules.php? $\mathrm{Na}$ me $=$ News $\&$ file $=$ article $\&$ sid $=184$. 
39- Univision . Disponible : Http://www.univision.com /content/content.jhtml:jsessionid=mvyjxajj4ffmqcwiaa4sfflkzaafgiwc? Cid-405538\&pagenum=4. 11 de Febrero 2005.

40- Wallace Anthony. Culture and Personality.1968..Random House.Toronto, Canada.

41- Warner Jennifer 2004.Disponible. http://my.webmd.com/ content/article/88/100087.htm 8 de Marzo 2005.

42- Yucesan Aydin , Oney Banu. (April 19-20 , 2000 ) . Literacy Development in Two Languages : Cognitive and Sociocultural Dimensions of Cross-Language Transfer.

Trabajo presentado en simposio: A research symposium on high standards in reading for students from diverse language groups : research, practice \& policy. Washington, DC U.S. Disponible: www. ncela.gwu.edu/pubs/symposia/reading/4oney.pdf

Recibido: 19/09/06 Aprobado: 30/04/07 